\title{
On Some I-Convergent Double Sequence Spaces Defined by a Modulus Function
}

\author{
Vakeel. A. Khan, Nazneen Khan \\ Department of Mathematics, Aligarh Muslim University, Aligarh, India \\ Email: vakhanmaths@gmail.com,nazneen4maths@gmail.com
}

Received February 15, 2013; revised March 17, 2013; accepted March 26, 2013

Copyright (C) 2013 Vakeel. A. Khan, Nazneen Khan. This is an open access article distributed under the Creative Commons Attribution License, which permits unrestricted use, distribution, and reproduction in any medium, provided the original work is properly cited.

\begin{abstract}
In 2000, Kostyrko, Salat, and Wilczynski introduced and studied the concept of $I$-convergence of sequences in metric spaces where I is an ideal. The concept of I-convergence has a wide application in the field of Number Theory, trigonometric series, summability theory, probability theory, optimization and approximation theory. In this article we introduce the double sequence spaces ${ }_{2} C_{0}^{I}(f),{ }_{2} C^{I}(f)$ and ${ }_{2} l_{\infty}^{I}(f)$ for a modulus function $f$ and study some of the properties of these spaces.
\end{abstract}

Keywords: Ideal; Filter; Modulus Function; Lipschitz Function; I-Convergence Field; I-Convergent; Monotone and Solid Double Sequence Spaces

\section{Introduction}

The notion of $I$-Convergence is a generalization of the concept statistical convergence which was first introduced by H. Fast [1] and later on studied by J. A. Fridy $[2,3]$ from the sequence space point of view and linked it with the summability theory. At the initial stage I-Convergence was studied by Kostyrko, Salat and Wilezynski [4]. Further it was studied by Salat, Tripathy, Ziman [5] and Demirci [6]. Throughout a double sequence is denoted by $x=\left(x_{i j}\right)$. Also a double sequence is a double infinite array of elements $x_{k l} \in \mathbb{R}$ for all $k, l \in \mathbb{N}$. The inital works on double sequences is found in Bromwich [7], Basarir and Solancan [8] and many others.

\section{Definitions and Preliminaries}

Throughout the article IN,IR, $\not \subset$ and $\omega$ denotes the set of natural, real, complex numbers and the class of all sequences respectively.

Let $X$ be a non empty set. A set $I \subseteq 2^{X}\left(2^{X}\right.$ denoting the power set of $X$ ) is said to be an ideal if $I$ is additive i.e $A, B \in I \Rightarrow A \cup B \in I$ and hereditary i.e. $A \in I, B \subseteq A \Rightarrow B \in I$.

A non-empty family of sets $£(I) \subseteq 2^{X}$ is said to be filter on $X$ if and only if $\Phi \notin £(I)$, for $A, B \in £(I)$ we

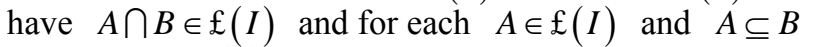
implies $B \subseteq \mathfrak{f}(I)$.
An Ideal $I \subseteq 2^{X}$ is called non-trivial if $I \neq 2^{X}$.

A non-trivial ideal $I \subseteq 2^{X}$ is called admissible if $\{\{x\}: x \in X\} \subseteq I$.

A non-trivial ideal $I$ is maximal if there cannot exist any non-trivial ideal $J \neq I$ containing $I$ as a subset.

For each ideal $I$, there is a filter $£(I)$ corresponding to $I$.

i.e. $£(I)=\left\{K \subseteq N: K^{c} \in I\right\}$, where $K^{c}=N-K$.

The idea of modulus was structured in 1953 by Nakano (See [9]).

A function $f:[0, \infty) \rightarrow[0, \infty)$ is called a modulus if

(1) $f(t)=0$ if and only if $t=0$,

(2) $f(t+u) \leq f(t)+f(u)$ for all $t, u \geq 0$,

(3) $f$ is nondecreasing, and

(4) $f$ is continuous from the right at zero.

Ruckle [10] used the idea of a modulus function $f$ to construct the sequence space

$$
X(f)=\left\{x=\left(x_{k}\right): \sum_{k=1}^{\infty} f\left(\left|x_{k}\right|\right)<\infty\right\} .
$$

This space is an FK space, and Ruckle[10] proved that the intersection of all such $X(f)$ spaces is $\phi$, the space of all finite sequences.

The space $X(f)$ is closely related to the space $l_{1}$ which is an $X(f)$ space with $f(x)=x$ for all real $x \geq 0$. Thus Ruckle [11] proved that, for any modulus $f$. 


$$
X(f) \subset l_{1} \text { and } X(f)^{\alpha}=l_{\infty}
$$

where

$$
X(f)^{\alpha}=\left\{y=\left(y_{k}\right) \in \omega: \sum_{k=1}^{\infty} f\left(\left|y_{k} x_{k}\right|\right)<\infty\right\}
$$

The space $X(f)$ is a Banach space with respect to the norm

$$
\|x\|=\sum_{k=1}^{\infty} f\left(\left|x_{k}\right|\right)<\infty \quad(\text { See }[10]) .
$$

Spaces of the type $X(f)$ are a special case of the spaces structured by B. Gramsch in [12]. From the point of view of local convexity, spaces of the type $X(f)$ are quite pathological. Therefore symmetric sequence spaces, which are locally convex have been frequently studied by D. J. H. Garling [13,14], G. Kothe [15] and W. H. Ruckle $[10,16]$.

Definition 2.1. A sequence space $E$ is said to be solid or normal if $\left(x_{i j}\right) \in E$ implies $\left(\alpha_{i j} x_{i j}\right) \in E$ for all sequence of scalars $\left(\alpha_{i j}\right)$ with $\left|\alpha_{i j}\right|<1$ for all $i, j \in I N$ (see [17])

Definition 2.2. Let

K

$=\left\{\left(n_{i}, k_{j}\right): i, j \in I N ; n_{1}<n_{2}<n_{3}<\cdots\right.$ and $\left.k_{1}<k_{2}<k_{3} \cdots\right\}$

$\subseteq I N \times I N$

and $E$ be a double sequence space. A $K$-step space of $E$ is a sequence space

$$
\lambda_{K}^{E}=\left\{\left(\alpha_{i j} x_{i j}\right):\left(x_{i j}\right) \in E\right\} .
$$

Definition 2.3. A cannonical preimage of a sequence $\left(x_{n_{i}, k_{j}}\right) \in E$ is a sequence $\left(b_{n, k}\right) \in E$ defined as follows

$$
b_{n, k}=\left\{\begin{array}{l}
a_{n, k}, \text { for } n, k \in K, \\
0, \quad \text { otherwise. }
\end{array} \quad(\text { see [18]). }\right.
$$

Definition 2.4. A sequence space $E$ is said to be monotone if it contains the cannonical preimages of all its stepspaces (see [19]).

Definition 2.5. A sequence space $E$ is said to be convergence free if $\left(y_{i j}\right) \in E$, whenever $\left(x_{i j}\right) \in E$ and $x_{i j}=0$ implies $y_{i j}=0$.

Definition 2.6. A sequence space $E$ is said to be a sequence algebra if $\left(x_{i j} y_{i j}\right) \in E$ whenever $\left(x_{i j}\right) \in E\left(y_{i j}\right) \in E$.

Definition 2.7. A sequence space $E$ is said to be symmetric if $\left(x_{\pi(i) \pi(j)}\right) \in E$ whenever $\left(x_{i j}\right) \in E$ where $\pi(i)$ and $\pi(j)$ is a permutation on $N$.

Definition 2.8. A sequence $\left(x_{i j}\right) \in \omega$ is said to be
$I$-convergent to a number $L$ if for every $\epsilon>0$. $\left\{(i, j) \in I N \times I N:\left|x_{i j}-L\right| \geq \epsilon\right\} \in I$. In this case we write $I-\lim x_{i j}=L$.

The space $c^{I}$ of all $I$-convergent sequences to $L$ is given by

$$
\begin{gathered}
c^{I}=\left\{\left(x_{i j}\right) \in \omega:\left\{(i, j) \in I N \times I N:\left|x_{i j}-L\right| \geq \epsilon\right\} \in I,\right. \\
\quad \text { for some } L \in \mathcal{Q}\}
\end{gathered}
$$

Definition 2.9. A sequence $(x)_{i j} \in \omega$ is said to be $I$-null if $L=0$. In this case we write $I$-lim $x_{i j}=0$.

Definition 2.10. A sequence $(x)_{i j} \in \omega$ is said to be $I$-cauchy if for every $\epsilon>0$ there exists a number $m=m(\epsilon)$ and $n=n(\epsilon)$ such that

$\left\{(i, j) \in I N \times I N:\left|x_{i j}-x_{m n}\right| \geq \epsilon\right\} \in I$.

Definition 2.11. A sequence $(x)_{i j} \in \omega$ is said to be $I$-bounded if there exists $M>0$ such that $\left\{(i, j) \in I N \times I N:\left|x_{i j}\right|>M\right\} \in I$

Definition 2.12. A modulus function $f$ is said to satisfy $\Delta_{2}$ condition if for all values of $u$ there exists a constant $K>0$ such that $f(L u) \leq K L f(u)$ for all values of $L>1$.

Definition 2.13. Take for $I$ the class $I_{f}$ of all finite subsets of $I N$. Then $I_{f}$ is a non-trivial admissible ideal and $I_{f}$ convergence coincides with the usual convergence with respect to the metric in $X$ (see [4]).

Definition 2.14. For $I=I_{\delta}$ and $A \subset I N$ with $\delta(A)=0$ respectively. $I_{\delta}$ is a non-trivial admissible ideal, $I_{\delta}$-convergence is said to be logarithmic statistical convergence (see [4]).

Definition 2.15. A map $\hbar$ defined on a domain $D \subset X$ i.e. $\hbar: D \subset X \rightarrow I R$ is said to satisfy Lipschitz condition if $|\hbar(x)-\hbar(y)| \leq K|x-y|$ where $K$ is known as the Lipschitz constant. The class of $K$-Lipschitz functions defined on $D$ is denoted by $\hbar \in(D, K)$ (see [20]).

Definition 2.16. A convergence field of $I$-convergence is a set

$$
F(I)=\left\{x=\left(x_{k}\right) \in l_{\infty} \text { : there exists } I-\lim x \in I R\right\} .
$$

The convergence field $F(I)$ is a closed linear subspace of $l_{\infty}$ with respect to the supremum norm, $F(I)=l_{\infty} \cap c^{I} \quad$ (See [5]).

Define a function $\hbar: F(I) \rightarrow I R$ such that $\hbar(x)=I-\lim x$, for all $x \in F(I)$, then the function $\hbar: F(I) \rightarrow I R$ is a Lipschitz function (see [20]). (c.f $[18,20-30]$ )

Throughout the article $l_{\infty}, c^{I}, c_{0}^{I}, m^{I}$ and $m_{0}^{I}$ represent the bounded, $I$-convergent, $I$-null, bounded $I$-convergent and bounded $I$-null sequence spaces respectively.

In this article we introduce the following classes of sequence spaces. 
${ }_{2} c^{I}(f)=\left\{\left(x_{i j}\right) \in \omega: I-\lim f\left(\left|x_{i j}\right|\right)=L\right.$ for some $\left.L\right\} \in I$

${ }_{2} c_{0}^{I}(f)=\left\{\left(x_{i j}\right) \in \omega: I-\lim f\left(\left|x_{i j}\right|\right)=0\right\} \in I$

${ }_{2} l_{\infty}^{I}(f)=\left\{\left(x_{i j}\right) \in \omega: \sup _{i j} f\left(\left|x_{i j}\right|\right)<\infty\right\} \in I$

We also denote by

$$
{ }_{2} m^{I}(f)={ }_{2} c^{I}(f) \cap{ }_{2} l_{\infty}(f)
$$

and

$$
{ }_{2} m_{0}^{I}(f)={ }_{2} c_{0}^{I}(f) \cap{ }_{2} l_{\infty}(f)
$$

The following Lemmas will be used for establishing some results of this article.

Lemma (1) Let $E$ be a sequence space. If $E$ is solid then $E$ is monotone.

Lemma (2) Let $K \in £(I)$ and $M \subseteq N$. If $M \notin I$, then $M \cap N \notin I$

Lemma (3) If $I \subset 2^{N}$ and $M \subseteq N$. If $M \notin I$, then $M \cap N \notin I$.

\section{Main Results}

Theorem 3.1. For any modulus function $f$, the classes of sequences ${ }_{2} c^{I}(f),{ }_{2} c_{0}^{I}(f),{ }_{2} m^{I}(f)$ and ${ }_{2} m_{0}^{I}(f)$ are linear spaces.

Proof: We shall prove the result for the space ${ }_{2} c^{I}(f)$.

The proof for the other spaces will follow similarly.

Let $\left(x_{i j}\right),\left(y_{i j}\right) \in{ }_{2} c^{I}(f)$ and let $\alpha, \beta$ be scalars. Then

$$
\begin{aligned}
& I-\lim f\left(\left|x_{i j}-L_{1}\right|\right)=0, \text { for some } L_{1} \in c \\
& I-\lim f\left(\left|y_{i j}-L_{2}\right|\right)=0, \text { for some } L_{2} \in c
\end{aligned}
$$

That is for a given $\epsilon>0$, we have

$$
\begin{aligned}
& A_{1}=\left\{(i, j) \in I N \times I N: f\left(\left|x_{i j}-L_{1}\right|\right)>\frac{\epsilon}{2}\right\} \in I, \\
& A_{2}=\left\{(i, j) \in I N \times I N: f\left(\left|y_{i j}-L_{2}\right|\right)>\frac{\epsilon}{2}\right\} \in I .
\end{aligned}
$$

Since $f$ is a modulus function, we have

$$
\begin{aligned}
& f\left(\left|\left(\alpha x_{i j}+\beta y_{i j}\right)-\left(\alpha L_{1}+\beta L_{2}\right)\right|\right) \\
& \leq f\left(|\alpha|\left|x_{i j}-L_{1}\right|\right)+f\left(|\beta|\left|y_{i j}-L_{2}\right|\right) \\
& \leq f\left(\left|x_{i j}-L_{1}\right|\right)+f\left(\left|y_{i j}-L_{2}\right|\right)
\end{aligned}
$$

Now, by (1) and (2),

$$
\begin{aligned}
& \left\{i, j \in N: f\left(\left|\left(\alpha x_{i j}+\beta y_{i j}\right)-\left(\alpha L_{1}+\beta L_{2}\right)\right|\right)>\epsilon\right\} \\
& \subset A_{1} \cup A_{2} .
\end{aligned}
$$

Therefore $\left(\alpha x_{i j}+\beta y_{i j}\right) \in{ }_{2} c^{I}(f)$

Hence ${ }_{2} c^{I}(f)$ is a linear space.

Theorem 3.2. A sequence $x=\left(x_{i j}\right) \in{ }_{2} m^{I}(f)$ is I-convergent if and only if for every $\epsilon>0$ there exists $I_{\epsilon}, J_{\epsilon} \in I N$ such that

$$
\left\{(i, j) \in I N \times I N: f\left(\left|x_{i j}-x_{I_{\epsilon}, J_{\epsilon}}\right|\right)<\epsilon\right\} \in{ }_{2} m^{I}(f)
$$

Proof: Suppose that $L=I-\lim x$. Then

$$
B_{\epsilon}=\left\{(i, j) \in I N \times I N:\left|x_{i j}-L\right|<\frac{\epsilon}{2}\right\} \in{ }_{2} m^{I}(f)
$$

For all $\epsilon>0$.

Fix an $I_{\epsilon}, J_{\epsilon} \in B_{\epsilon}$. Then we have

$$
\left|X_{I_{\epsilon} J_{\epsilon}}-x_{i j}\right| \leq\left|X_{I_{\epsilon} J_{\epsilon}}-L\right|+\left|L-X_{i j}\right|<\frac{\epsilon}{2}+\frac{\epsilon}{2}=\epsilon
$$

which holds for all $i, j \in B_{\epsilon}$.

Hence $\left\{(i, j) \in I N \times I N: f\left(\left|x_{i j}-x_{I_{\epsilon} J_{\epsilon}}\right|\right)<\epsilon\right\} \in{ }_{2} m^{I}(f)$.

Conversely, suppose that

$$
\left\{(i, j) \in I N \times I N: f\left(\left|x_{i j}-x_{I_{\epsilon} J_{\epsilon}}\right|\right)<\epsilon\right\} \in{ }_{2} m^{I}(f) .
$$

That is $\left\{(i, j) \in I N \times I N:\left(\left|x_{i j}-x_{I_{\epsilon} J_{\epsilon}}\right|\right)<\epsilon\right\} \in{ }_{2} m^{I}(f)$ for all $\epsilon>0$. Then the set

$$
\begin{aligned}
& { }_{2} C_{\epsilon}=\left\{(i, j) \in I N \times I N: x_{i j} \in\left[x_{I_{\epsilon} J_{\epsilon}}-\epsilon, x_{I_{\epsilon} J_{\epsilon}}+\epsilon\right]\right\} \\
& { }_{2} m^{I}(f) \text { for all } \epsilon>0 .
\end{aligned}
$$

Let $N_{\epsilon}=\left[x_{I_{\epsilon} J_{\epsilon}}-\epsilon, x_{I_{\epsilon} J_{\epsilon}}+\epsilon\right]$. If we fix an $\epsilon>0$ then we have ${ }_{2} C_{\epsilon} \in{ }_{2} m^{I}(f)$ as well as ${ }_{2} C_{\frac{\epsilon}{2}} \in{ }_{2} m^{I}(f)$. Hence ${ }_{2} C_{\epsilon} \cap{ }_{2} C_{\epsilon} \in{ }_{2} m^{I}(f)$. This implies that

$$
N_{\epsilon} \cap N_{\frac{\epsilon}{2}} \neq \phi
$$

that is

$$
\left\{(i, j) \in I N \times I N: x_{i j} \in N\right\} \in{ }_{2} m^{I}(f)
$$

that is

$$
\operatorname{diam} N \leq \operatorname{diam} N_{\epsilon}
$$

where the diam of $N$ denotes the length of interval $N$.

In this way, by induction we get the sequence of closed intervals

$$
N_{\epsilon}=I_{0} \supseteq I_{1} \supseteq \cdots \supseteq I_{i j} \supseteq \cdots .
$$

with the property that $\operatorname{diam} I_{i j} \leq \frac{1}{2} \operatorname{diam} I_{(i-1)(j-1)}$ for $(i, j=2,3,4, \cdots)$ and

$\left\{(i, j) \in I N \times I N: x_{i j} \in I_{i j}\right\} \in{ }_{2} m^{I}(f)$ for

$(i, j=1,2,3,4, \cdots)$. 
Then there exists a $\xi \in \bigcap I_{i j}$ where $i, j \in I N$ such that $\xi=I-\lim x$. So that $f(\xi)=I-\lim f(x)$, that is $L=I-\lim f(x)$.

Theorem 3.3. Let $f$ and $g$ be modulus functions that satisfy the $\Delta_{2}$-condition.If $X$ is any of the spaces ${ }_{2} C^{I},{ }_{2} C_{0}^{I},{ }_{2} m^{I}$ and ${ }_{2} m_{0}^{I}$ etc, then the following assertions hold.

(i) $X(g) \subseteq X(f \cdot g)$,

(ii) $X(f) \cap X(g) \subseteq X(f+g)$.

Proof: (i) Let $\left(x_{i j}\right) \in{ }_{2} c_{0}^{I}(g)$. Then

$$
I-\lim _{i j} g\left(\left|X_{i j}\right|\right)=0
$$

Let $\epsilon>0$ and choose $\delta$ with $0<\delta<1$ such that $f(t)<\epsilon$ for $0<t<\delta$.

Write $y_{i j}=g\left(\left|x_{i j}\right|\right)$ and consider

$\lim _{i j} f\left(y_{i j}\right)=\lim _{i j} f\left(y_{i j}\right)_{y_{i j}<\delta}+\lim _{i j} f\left(y_{i j}\right)_{y_{i j}>\delta}$

We have

$$
\lim _{i j} f\left(y_{i j}\right) \leq f(2) \lim _{i j}\left(y_{i j}\right)
$$

For $y_{i j}>\delta$, we have $y_{i j}<\frac{y_{i j}}{\delta}<1+\frac{y_{i j}}{\delta}$. Since $f$ is non-decreasing, it follows that

$$
f\left(y_{i j}\right)<f\left(1+\frac{y_{i j}}{\delta}\right)<\frac{1}{2} f(2)+\frac{1}{2} f\left(\frac{2 y_{i j}}{\delta}\right)
$$

Since $f$ satisfies the $\Delta_{2}$-condition, we have

$$
f\left(y_{i j}\right)<\frac{1}{2} K \frac{y_{i j}}{\delta} f(2)+\frac{1}{2} K \frac{y_{i j}}{\delta} f(2)=K \frac{y_{i j}}{\delta} f(2)
$$

Hence

$$
\lim _{i j} f\left(y_{i j}\right) \leq \max (1, K) \delta^{-1} f(2) \lim _{i j}\left(y_{i j}\right) .
$$

From (4), (5) and (6), we have $\left(x_{i j}\right) \in{ }_{2} C_{0}^{I}(f \cdot g)$.

Thus ${ }_{2} C_{0}^{I}(g) \subseteq{ }_{2} C_{0}^{I}(f \cdot g)$. The other cases can be proved similarly.

(ii) Let $\left(x_{i j}\right) \in{ }_{2} c_{0}^{I}(f) \cap_{2} c_{0}^{I}(g)$. Then

$$
\begin{aligned}
I-\lim _{i j} f\left(\left|x_{i j}\right|\right) & =0 \text { and } I-\lim _{i j} g\left(\left|x_{i j}\right|\right)=0 \\
\lim _{i j}(f+g)\left(\left|x_{i j}\right|\right) & =\lim _{i j} f\left(\left|x_{i j}\right|\right)+g\left(\left|x_{i j}\right|\right) \\
& =\lim _{i j} f\left(\left|x_{i j}\right|\right)+\lim _{i j} g\left(\left|x_{i j}\right|\right)=0
\end{aligned}
$$

Therefore

$$
\lim _{i j}(f+g)\left(\left|x_{i j}\right|\right)=0
$$

which implies $\left(x_{i j}\right) \in X(f+g)$, that is

$$
X(f) \cap X(g) \subseteq X(f+g) \text {. }
$$

Corollary 3.4. $X \subseteq X(f)$ for $X={ }_{2} C^{I},{ }_{2} C_{0}^{I},{ }_{2} m^{I}$ and ${ }_{2} m_{0}^{I}$.
Proof: The result can be easily proved using $f(x)=x$ for $x=\left(x_{i j}\right) \in X$.

Theorem 3.5. The spaces ${ }_{2} c_{0}^{I}(f)$ and ${ }_{2} m_{0}^{I}(f)$ are solid and monotone.

Proof: We shall prove the result for ${ }_{2} C_{0}^{I}(f)$. Let $x_{i j} \in{ }_{2} c_{0}^{I}(f)$. Then

$$
I-\lim _{i j} f\left(\left|x_{i j}\right|\right)=0
$$

Let $\left(\alpha_{i j}\right)$ be a sequence of scalars with $\left|\alpha_{i j}\right| \leq 1$ for all $i, j \in I N$. Then we have

$$
\begin{aligned}
& I-\lim _{i j} f\left(\left|\alpha_{i j} x_{i j}\right|\right) \leq I-\lim _{i j} f\left(\left|\alpha_{i j}\right|\left|x_{i j}\right|\right) \\
& =\left|\alpha_{i j}\right| I-\lim _{i j} f\left(\left|x_{i j}\right|\right)=0 \\
& I-\lim _{i j} f\left(\left|\alpha_{i j} x_{i j}\right|\right)=0 \text { for all } i, j \in I N .
\end{aligned}
$$

which implies that $\alpha_{i j} x_{i j} \in{ }_{2} c_{0}^{I}(f)$.

Therefore the space ${ }_{2} c_{0}^{I}(f)$ is solid. The space ${ }_{2} c_{0}^{I}(f)$ is monotone follows from Lemma (1). For ${ }_{2} m_{0}^{I}(f)$ the result can be proved similarly.

Theorem 3.6. The spaces ${ }_{2} C^{I}(f)$ and ${ }_{2} m^{I}(f)$ are neither solid nor monotone in general.

Proof: Here we give a counter example.

Let $I=I_{\delta}$ and $f(x)=x^{2}$ for all $x \in[0, \infty)$. Consider the $K$-step space $X_{K}(f)$ of $X$ defined as follows, Let $\left(x_{i j}\right) \in X$ and let $\left(y_{i j}\right) \in X_{K}$ be such that

$$
\left(y_{i j}\right)=\left\{\begin{array}{l}
\left(x_{i j}\right), \text { if } i, j \text { is even, } \\
0, \quad \text { otherwise. }
\end{array}\right.
$$

Consider the sequence $\left(x_{i j}\right)$ defined by $\left(x_{i j}\right)=1$ for all $i, j \in N$.

Then $\left(x_{i j}\right) \in{ }_{2} c^{I}(f)$ but its $K$-stepspace preimage does not belong to ${ }_{2} C^{I}(f)$. Thus ${ }_{2} C^{I}(f)$ is not monotone. Hence ${ }_{2} c^{I}(f)$ is not solid.

Theorem 3.7. The spaces ${ }_{2} c^{I}(f)$ and ${ }_{2} c_{0}^{I}(f)$ are sequence algebras.

Proof: We prove that ${ }_{2} c_{0}^{I}(f)$ is a sequence algebra.

Let $\left(x_{i j}\right),\left(y_{i j}\right) \in{ }_{2} c_{0}^{I}(f)$. Then

$$
I-\lim f\left(\left|x_{i j}\right|\right)=0
$$

and

$$
I-\lim f\left(\left|y_{i j}\right|\right)=0
$$

Then we have

$$
I-\lim f\left(\left|\left(x_{i j} \cdot y_{i j}\right)\right|\right)=0
$$

Thus $\left(x_{i j} \cdot y_{i j}\right) \in{ }_{2} c_{0}^{I}(f)$ is a sequence algebra.

For the space ${ }_{2} c^{I}(f)$, the result can be proved similarly.

Theorem 3.8. The spaces ${ }_{2} c^{I}(f)$ and ${ }_{2} c_{0}^{I}(f)$ are not convergence free in general.

Proof: Here we give a counter example. 
Let $I=I_{f}$ and $f(x)=x^{3}$ for all $x \in[0, \infty)$. Consider the sequence $\left(x_{i j}\right)$ and $\left(y_{i j}\right)$ defined by $x_{i j}=\frac{1}{i+j}$ and $y_{i j}=i+j$ for all $i, j \in I N$

Then $\left(x_{i j}\right) \in c^{I}(f)$ and $c_{0}^{I}(f)$, but $\left(y_{i j}\right) \notin c^{I}(f)$ and $c_{0}^{I}(f)$.

Hence the spaces $c^{I}(f)$ and $c_{0}^{I}(f)$ are not convergence free.

Theorem 3.9. If $I$ is not maximal and $I \neq I_{f}$, then the spaces ${ }_{2} c^{I}(f)$ and ${ }_{2} c_{0}^{I}(f)$ are not symmetric.

Proof: Let $A \in I$ be infinite and $f(x)=x$ for all $x \in[0, \infty)$.

$$
x_{i j}=\left\{\begin{array}{l}
1, \text { for } i, j \in A, \\
0, \text { otherwise. }
\end{array}\right.
$$

Then by Lemma (3) we have $x_{i j} \in{ }_{2} c_{0}^{I}(f) \subset{ }_{2} c^{I}(f)$.

Let $K \subset I N$ be such that $K \notin I$ and $I N-K \notin I$.

Let $\phi: K \rightarrow A$ and $\psi: I N-K \rightarrow I N-A$ be bijections, then the map $\pi: I N \rightarrow I N$ defined by

$$
\pi(i j)=\left\{\begin{array}{l}
\phi(i j), \text { for } i, j \in K, \\
\psi(i j), \text { otherwise }
\end{array}\right.
$$

is a permutation on $I N$, but $x_{\pi(i j)} \notin{ }_{2} c^{I}(f)$ and $x_{\pi(i j)} \notin{ }_{2} c_{0}^{I}(f)$.

Hence ${ }_{2} c_{0}^{I}(f)$ and ${ }_{2} c^{I}(f)$ are not symmetric.

Theorem 3.10. Let $f$ be a modulus function. Then ${ }_{2} C_{0}^{I}(f) \subset{ }_{2} C^{I}(f) \subset{ }_{2} l_{\infty}^{I}(f)$ and the inclusions are proper.

Proof: The inclusion ${ }_{2} c_{0}^{I}(f) \subset{ }_{2} c^{I}(f)$ is obvious.

Let $x=x_{i j} \in{ }_{2} C^{I}(f)$. Then there exists $L \in C$ such that

$$
I-\lim f\left(\left|x_{i j}-L\right|\right)=0 .
$$

We have $f\left(\left|x_{i j}\right|\right) \leq \frac{1}{2} f\left(\left|x_{i j}-L\right|\right)+f \frac{1}{2}(|L|)$.

Taking the supremum over $i$ and $j$ on both sides we get $x_{i j} \in{ }_{2} l_{\infty}(f)$.

Next we show that the inclusion is proper.

(i) ${ }_{2} c_{0}^{I}(f) \subset{ }_{2} c^{I}(f)$

Let $x=\left(x_{i j}\right) \in{ }_{2} c^{I}(f)$ then $I-\lim f\left(\left|x_{i j}\right|\right)=L$ for some $L(\neq 0) \in C$, which implies $x \notin{ }_{2} c_{0}^{I}(f)$. Hence the inclusion is proper.

(ii) ${ }_{2} c^{I}(f) \subset{ }_{2} l_{\infty}^{I}(f)$. Let $x=\left(x_{i j}\right) \in{ }_{2} l_{\infty}^{I}(f)$ then

$$
\begin{aligned}
& I-\lim f\left(\left|x_{i j}\right|\right)<\infty \\
& I-\lim f\left(\left|x_{i j}-L+L\right|\right)<\infty \\
& I-\lim f\left(\left|x_{i j}-L\right|\right)+I-\lim f(|L|)<\infty \\
& I-\lim f\left(\left|x_{i j}-L\right|\right)<\infty \\
& I-\lim f\left(\left|x_{i j}-L\right|\right) \neq 0
\end{aligned}
$$

Therefore $x \notin{ }_{2} c^{I}(f)$, and hence the inclusion is proper.

Theorem 3.11. The function $\hbar:{ }_{2} m^{I}(f) \rightarrow I R$ is the Lipschitz function, where ${ }_{2} m^{I}(f)={ }_{2} c^{I}(f) \cap{ }_{2} l_{\infty}(f)$, and hence uniformly continuous.

Proof: Let $x, y \in{ }_{2} m^{I}(f), x \neq y$. Then the sets

$$
\begin{aligned}
& A_{x}=\left\{(i, j) \in I N \times I N:\left|x_{i j}-\hbar(x)\right| \geq\|x-y\|\right\} \in I, \\
& A_{y}=\left\{(i, j) \in I N \times I N:\left|y_{i j}-\hbar(y)\right| \geq\|x-y\|\right\} \in I .
\end{aligned}
$$

Thus the sets,

$B_{x}=\left\{(i, j) \in I N \times I N:\left|x_{i j}-\hbar(x)\right|<\|x-y\|\right\} \in{ }_{2} m^{I}(f)$,

$B_{y}=\left\{(i, j) \in I N \times I N:\left|y_{i j}-\hbar(y)\right|<\|x-y\|\right\} \in{ }_{2} m^{I}(f)$.

Hence also $B_{x} \cap B_{y} \in{ }_{2} m^{I}(f)$, so that $B \neq \phi$.

Now taking $i, j$ in $B$,

$$
\begin{aligned}
& |\hbar(x)-\hbar(y)| \\
& \leq\left|\hbar(x)-x_{i j}\right|+\left|x_{i j}-y_{i j}\right|+\left|y_{i j}-\hbar(y)\right| \\
& \leq 3|| x-y \| .
\end{aligned}
$$

Thus $\hbar$ is a Lipschitz function. For ${ }_{2} m_{0}^{I}(f)$ the result can be proved similarly.

Theorem 3.12. If $x, y \in{ }_{2} m^{I}(f)$, then $(x \cdot y) \in{ }_{2} m^{I}(f)$ and $\hbar(x y)=\hbar(x) \hbar(y)$.

Proof: For $\epsilon>0$

$$
\begin{aligned}
& B_{x}=\left\{(i, j) \in I N \times I N:\left|x_{i j}-\hbar(x)\right|<\epsilon\right\} \in{ }_{2} m^{I}(f), \\
& B_{y}=\left\{(i, j) \in I N \times I N:\left|y_{i j}-\hbar(y)\right|<\epsilon\right\} \in{ }_{2} m^{I}(f) .
\end{aligned}
$$

Now,

$$
\begin{aligned}
& \left|x_{i j} y_{i j}-\hbar(x) \hbar(y)\right| \\
& =\left|x_{i j} y_{i j}-x_{i j} \hbar(y)+x_{i j} \hbar(y)-\hbar(x) \hbar(y)\right| \\
& \leq\left|x_{i j}\right|\left|y_{i j}-\hbar(y)\right|+|\hbar(y)|\left|x_{i j}-\hbar(x)\right|
\end{aligned}
$$

As ${ }_{2} m^{I}(f) \subseteq{ }_{2} l_{\infty}(f)$, there exists an $M \in I R$ such that $\left|x_{i j}\right|<M$ and $|\hbar(y)|<M$.

Using Equation (8) we get

$$
\left|x_{i j} y_{i j}-\hbar(x) \hbar(y)\right| \leq M \epsilon+M \epsilon=2 M \epsilon
$$

For all $i, j \in B_{x} \cap B_{y} \in{ }_{2} m^{I}(f)$. Hence $(x \cdot y) \in{ }_{2} m^{I}(f)$ and $\hbar(x y)=\hbar(x) \hbar(y)$.

For ${ }_{2} m_{0}^{I}(f)$ the result can be proved similarly.

\section{Acknowledgements}

The authors would like to record their gratitude to the reviewer for his careful reading and making some useful corrections which improved the presentation of the paper. 


\section{REFERENCES}

[1] H. Fast, "Sur la Convergence Statistique," Colloqium Mathematicum, Vol. 2, No. 1, 1951, pp. 241-244.

[2] J. A. Fridy, "On Statistical Convergence," Analysis, Vol. 5, 1985, pp. 301-313.

[3] J. A. Fridy, "Statistical Limit Points," Proceedings of American Mathematical Society, Vol. 11, 1993, pp. 11871192. doi:10.1090/S0002-9939-1993-1181163-6

[4] P. Kostyrko, T. Salat and W. Wilczynski, "I-Convergence," Real Analysis Exchange, Vol. 26, No. 2, 1999, pp. 193-200.

[5] T. Salat, B. C. Tripathy and M. Ziman, "On Some Properties of I-Convergence," Tatra Mountain Mathematical Publications, 2000, pp. 669-686.

[6] K. Demirci, "I-Limit Superior and Limit Inferior," $M a-$ thematical Communications, Vol. 6, 2001, pp. 165-172.

[7] T. J. I. Bromwich, "An Introduction to the Theory of Infinite Series,” MacMillan Co. Ltd., New York, 1965.

[8] M. Basarir and O. Solancan, "On Some Double Sequence Spaces," Journal of the Indian Academy of Mathematics, Vol. 21, No. 2, 1999, pp. 193-200.

[9] H. Nakano, "Concave Modulars," Journal of Mathematical Society, Japan, Vol. 5, No. 1, 1953, pp. 29-49. doi:10.2969/jmsj/00510029

[10] W. H. Ruckle, "On Perfect Symmetric BK-Spaces," Mathematische Annalen, Vol. 175, No. 2, 1968, pp. 121-126. doi:10.1007/BF01418767

[11] W. H. Ruckle, "FK-Spaces in Which the Sequence of Coordinate Vectors is Bounded," Canadian Journal of Mathematics, Vol. 25, No. 5, 1973, pp. 973-975. doi:10.4153/CJM-1973-102-9

[12] B. Gramsch, "Die Klasse Metrisher Linearer Raume L( $\varphi)$," Mathematische Annalen, Vol. 171, 1967, pp. 6178. doi:10.1007/BF01433094

[13] D. J. H. Garling, "On Symmetric Sequence Spaces,” Proceedings of London Mathematical Society, Vol. 16, 1966, pp. 85-106. doi:10.1112/plms/s3-16.1.85

[14] D. J. H. Garling, "Symmetric Bases of Locally Convex Spaces," Studia Mathematica, Vol. 30, No. 2, 1968, pp. 163-181.

[15] G. Kothe, "Topological Vector Spaces," Springer, Berlin, 1970.

[16] W. H. Ruckle, "Symmetric Coordinate Spaces and Symmetric Bases," Canadian Journal of Mathematics, Vol.
19, 1967, pp. 828-838. doi:10.4153/CJM-1967-077-9

[17] V. A. Khan and S. Tabassum, "On Some New Double Sequence Spaces of Invariant Means Defined by Orlicz Function," Communications, Faculty of Sciences, University of Ankara, Vol. 60, 2011, pp. 11-21.

[18] J. Singer, "Bases in Banach Spaces. 1," Springer, Berlin, 1970.

[19] M. Sen and S. Roy, "Some I-Convergent Double Classes of Sequences of Fuzzy Numbers Defined by Orlicz Functions," Thai Journal of Mathematics, Vol. 10, No. 4, 2013, pp. 1-10.

[20] I. J. Maddox, "Some Properties of Paranormed Sequence Spaces," Journal of the London Mathematical Society, Vol. 1, 1969, pp. 316-322.

[21] J. Connor and J. Kline, "On Statistical Limit Points and the Consistency of Statistical Convergence," Journal of Mathematical Analysis and Applications, Vol. 197, No. 2 , 1996, pp. 392-399. doi:10.1006/jmaa.1996.0027

[22] K. Dems, "On I-Cauchy Sequences," Real Analysis Exchange, Vol. 30, No. 1, 2005, pp. 123-128.

[23] M. Gurdal, "Some Types Of Convergence," Doctoral Dissertation, Sleyman Demirel University, Isparta, 2004.

[24] O. T. Jones and J. R. Retherford, "On Similar Bases in Barrelled Spaces," Proceedings of American Mathematical Society, Vol. 18, 1967, pp. 677-680. doi:10.1090/S0002-9939-1967-0217552-8

[25] P. K. Kamthan and M. Gupta, "Sequence Spaces and Series," Marcel Dekker Inc., New York, 1981.

[26] I. J. Maddox, "Elements of Functional Analysis," Cambridge University Press, Cambridge, 1970.

[27] I. J. Maddox, "Sequence Spaces Defined by a Modulus," Mathematical Proceedings of the Cambridge Philosophical Society, Vol. 100, 1986, pp. 161-166. doi:10.1017/S0305004100065968

[28] T. Salat, "On Statistically Convergent Sequences of Real Numbers," Mathematica Slovaca, Vol. 30, 1980, pp. 139. 150 .

[29] A. K. Vakeel and K. Ebadullah, "On Some I-Convergent Sequence Spaces Defined by a Modulus Function," Theory and Applications of Mathematics and Computer Science, Vol. 1, No. 2, 2011, pp. 22-30.

[30] A. Wilansky, "Functional Analysis," Blaisdell, New York, 1964. 